\title{
Iranian Registry of Crohn's and Colitis: study profile of first nation-wide inflammatory bowel disease registry in Middle East
}

\author{
Masoud M Malekzadeh ${ }^{1}$, Alireza Sima ${ }^{1}$, Sudabeh Alatab $^{1}$, Anahita Sadeghi ${ }^{1}$, Nasser Ebrahimi Daryani ${ }^{2}$, \\ Payman Adibi ${ }^{3}$, Iradj Maleki ${ }^{4}$, Hassan Vossoughinia ${ }^{5}$, Hafez Fakheri ${ }^{4}$, Abbas Yazdanbod ${ }^{6}$, Seyed Alireza Taghavi ${ }^{7}$, \\ Rahim Aghazadeh ${ }^{8}$, Mohammad Hassan Somi ${ }^{9}$, Kazem Zendedel $^{10,11}$, Homayoon Vahedi ${ }^{1}$, Reza Malekzadeh ${ }^{1}$ \\ ${ }^{1}$ Digestive Disease Research Center, Digestive Disease Research Institute, Shariati Hospital, Tehran University of Medical Sciences, Tehran; \\ ${ }^{2}$ Department of Gastroenterology, Imam Khomeini Hospital, Tehran University of Medical Sciences, Tehran; ${ }^{3}$ Gastroenterology Section, \\ Department of Internal Medicine, Isfahan University of Medical Sciences, Isfahan; ${ }^{4}$ Gut and Liver Research Center, Mazandaran University of \\ Medical Sciences, Sari; ${ }^{5}$ Department of Gastroenterology and Hepatology, Ghaem Hospital, Medical Faculty, Mash had University of Medical \\ Sciences, Mashhad; ${ }^{6}$ Department of Medicine, Ardabil University of Medical Science, Ardabil; ${ }^{7}$ Gastroenterohepatology Research Center, Shiraz \\ University of Medical Sciences, Shiraz; ${ }^{8}$ Gastroenterology and Liver Diseases Research Center, Research Institute for Gastroenterology and \\ Liver Diseases, Shahid Beheshti University of Medical Sciences, Tehran; ${ }^{9}$ Liver and Gastrointestinal Diseases Research Center, Tabriz University \\ of Medical Sciences, Tabriz; ${ }^{10}$ Ministry of Health and Medical Education, Tehran; ${ }^{11}$ Cancer Research Center, Cancer Institute of Iran, Tehran \\ University of Medical Sciences, Tehran, Iran
}

Background/Aims: A recent study revealed increasing incidence and prevalence of inflammatory bowel disease (IBD) in Iran. The Iranian Registry of Crohn's and Colitis (IRCC) was designed recently to answer the needs. We reported the design, methods of data collection, and aims of IRCC in this paper. Methods: IRCC is a multicenter prospective registry, which is established with collaboration of more than 100 gastroenterologists from different provinces of Iran. Minimum data set for IRCC was defined according to an international consensus on standard set of outcomes for IBD. A pilot feasibility study was performed on 553 IBD patients with a web-based questionnaire. The reliability of questionnaire evaluated by Cronbach's $\alpha$. Results: All sections of questionnaire had Cronbach's $\alpha$ of more than 0.6. In pilot study, 312 of participants (56.4\%) were male and mean age was 38 years (standard deviation $=12.8$ ) and 378 patients $(68.35 \%)$ had ulcerative colitis, 303 subjects $(54,7 \%)$ had college education and 358 patients (64.74\%) were of Fars ethnicity. We found that 68 (12.3\%), $44(7.9 \%)$, and 13 (2.3\%) of participants were smokers, hookah and opium users, respectively. History of appendectomy was reported in 58 of patients (10.48\%). The most common medication was 5-aminosalicylate (94.39\%). Conclusions: To the best of our knowledge, IRCC is the first national IBD registry in the Middle East and could become a reliable infrastructure for national and international research on IBD. IRCC will improve the quality of care of IBD patients and provide national information for policy makers to better plan for controlling IBD in Iran. (Intest Res 2019;17:330-339)

Key Words: Inflammatory bowel disease; Registry; Iran

Received December 3, 2018. Revised February 7, 2019.

Accepted February 8, 2019.

Correspondence to Homayoon Vahedi, Digestive Disease Research Center, Digestive Disease Research Institute, Shariati Hospital, Tehran University of Medical Sciences, North Kargar Ave., Tehran 14117-13135, Iran. Tel: +98-2182415104, Fax: +98-21-82415400, E-mail: homayoonvahedi@gmail.com ORCID Masoud M Malekzadeh (https://orcid.org/0000-0002-6792-3155), Homayoon Vahedi (https://orcid.org/0000-0002-9965-7242)

\section{INTRODUCTION}

Incidence rate of IBD is stabilizing in some developed countries; ${ }^{1-4}$ however, the incidence rate is increasing in developing countries such as Asia and Eastern Europe..$^{5-7}$ Recent nationwide report in Iran showed the increasing trend in incidence 
and prevalence of both UC and $\mathrm{CD}^{8}{ }^{8}$ The rising incidence and prevalence of IBD made an essence to investigate the risk factors and possible etiologies of this disease and necessitates further studies for better understanding of natural history, phenotype, treatment response, complication, and survival of the patients who suffer from IBD. Establishing a prospective IBD registry is one the best way to answer these questions. ${ }^{9}$

Iranian ministry of health have recently launched a program of supporting disease registries. ${ }^{10}$ Following this initiative, we established a multicenter national IBD registry named "Iranian Registry of Crohn's and Colitis (IRCC).” Each disease registry has its own limitation and strength and thereby could not answer all clinical or epidemiological questions related to that disease. ${ }^{11}$ Introducing the study design, data collection, and aims of IRCC enlighten the strengths and limitations and may help researchers to better interpret the results of this nationwide study in future.

The aim of IRCC is to study clinical phenotype, safety of treatment, pattern of care across country, risk of colorectal cancer, prognostic factors, complication, survival, incidence and prevalence of IBD in Iran. Another objective is to set up a blood and stool biobank of IBD patients which its protocol will be published in near future. In this report we described the study protocol, process of IRCC implementation and assessment of its feasibility in Iran.

\section{METHODS}

\section{Registry Setting and Organization}

IRCC is a multicenter prospective registry enrolling adult patients diagnosed as IBD. Patients are managed according to their routine care and no intervention from the registry team would be imposed. The registry comprises of referral centers of IBD located in the capital and all provincial centers of Iran.

IRCC was organized under the guidance of steering committee of 7 senior gastroenterologists from major gastroenterology departments and research centers of Iran. These members were selected based on their expertise and interest in research and care of IBD patients. One member of steering committee was selected as principal investigator and director of registry in order to supervise the executive team (Fig. 1) which consists of an executive manager, 2 registrars, a nurse as patient educator, an information technology (IT) technician and 2 researchers. Gastroenterologists who shared their cases with registry were considered as IRCC collaborators. The executive team had a time chart and objectives for each year which were

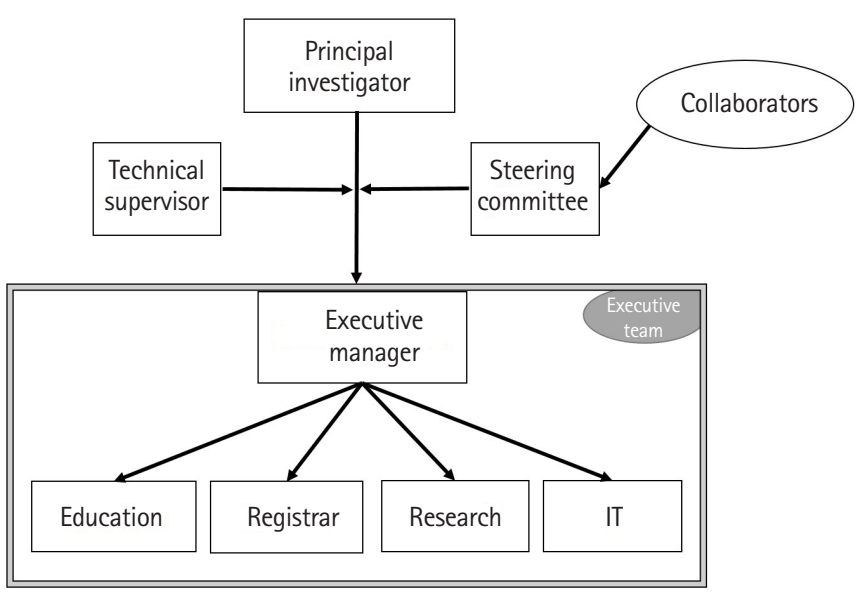

Fig. 1. The Iranian Registry of Crohn's and Colitis organization. IT, information technology.

followed in weekly regular meeting. An authorship guideline was approved by steering committee after receiving comments from all collaborators. The study was approved in Ethic Committee of Digestive Disease Research Institute of Tehran University of Medical Sciences. IRCC was funded by deputy of research of the Ministry of Health and Medical Education and was strongly supported for all logistics by the Iranian Association of Gastroenterology and Hepatology (IAGH).

In Iran, all IBD patients were served at provincial center rather than villages or small cities. IRCC has at least 1 center in each province. From different provinces, 449 gastroenterologists who visit IBD patients in public or private clinics have accepted to collaborate with IRCC. According to report from IAGH 517 gastroenterologists are presently working in Iran. Therefore, IRCC covers $87 \%$ of all GI centers around the country (Fig. 2, Supplementary Table 1). However, the exact participation rate could be calculated after finishing the registry process. Of different provinces of Iran, the most modernized and industrialized are Tehran, Razavi Khorasan, Fars, Isfahan, Mazandaran, East Azerbaijan, and Gilan in which the majority of gastroenterologists work and most of IBD patient were treated there. Distribution of gastroenterologists in Iran was shown in Supplementary Table 1 and Supplementary Fig. 1.

\section{Study Population}

Study population consisted of adult who were diagnosed as IBD by gastroenterologists in collaborating centers across the country. The exclusion criteria were set for patients who aged less than 18 years and for those who were unable or unwilling to provide informed consent. Patients with un-confirmed diagnosis also were excluded. 


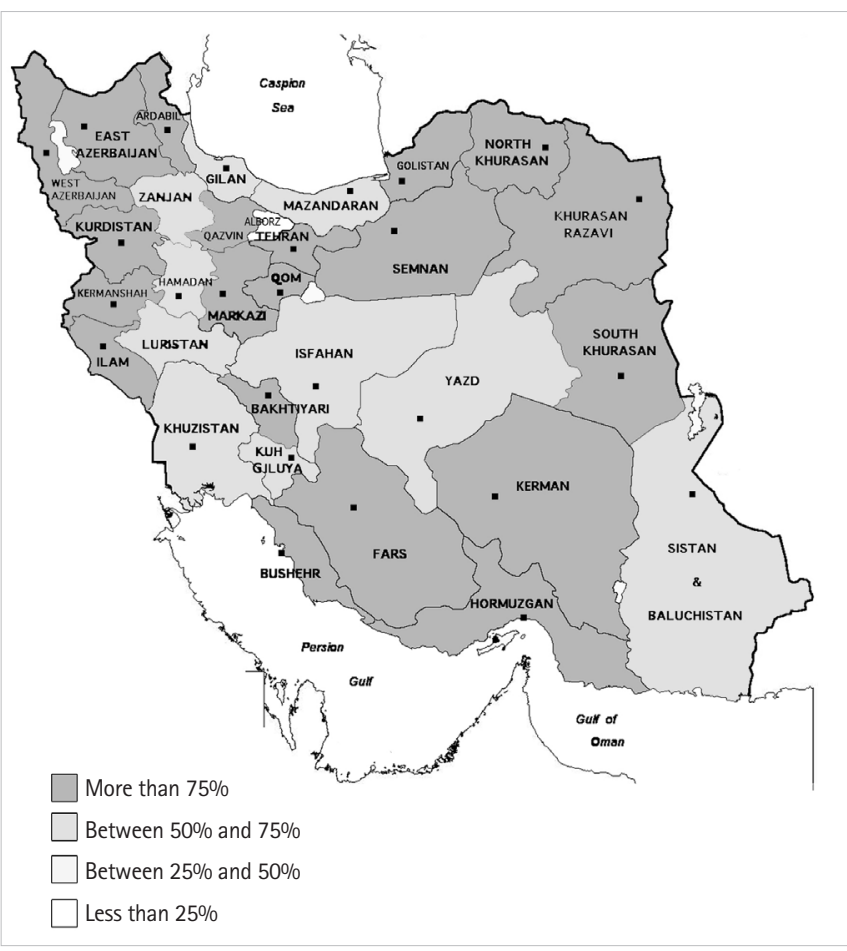

Fig. 2. Participation rate of gastroenterologists in different provinces of Iran with Iranian Registry of Crohn's and Colitis study.

\section{Disease Definition}

IBD is defined as combination of clinical, radiological, colonoscopic, and pathologic and classified to UC and CD according to international IBD guideline ${ }^{12}$ as below. This uniform criterion was attached as appendix to IRCC questionnaire and sent to all collaborating provincial centers.

\section{1) Clinical}

History of rectal bleeding or bloody mucus diarrhea, abdominal pain, vomiting, weight loss, fistulas, and fever. Finding such as pallor, cachexia, mild abdominal tenderness, perianal involvement (fissure, fistula, and abscess) in physical examination. Predominantly bloody diarrhea was in favor of UC. Abdominal pain, malnutrition, and perianal lesions were in favor of CD.

\section{2) Laboratory Tests}

Evidence of anemia and thrombocytosis in complete blood count, elevated ESR or CRP, negative stool exam and culture to rule out bacterial or parasitic infection and elevated level of fecal calprotectin. Anti-neutrophil cytoplasmic antibody and Anti-Saccharomyces cerevisiae antibody were in favor of UC and CD respectively.

\section{3) Colonoscopic}

Evidence of ulcers, aphthous lesions, inflammation, bleeding, stenosis, pseudo-polyps in colon. Capsule endoscopy and enteroscopy were used in cases of small bowel involvement when other modalities have been negative and IBD was strongly suspected. Continuous superficial colonic mucosal lesions, and spontaneous bleeding were in favor of UC. Patchy transmural asymmetric lesions mainly involving ileum and rightsided colon, deep fissures, cobblestone appearance, and longitudinal ulcers were in favor of CD.

\section{4) Imaging}

CT or magnetic resonance enterography were used to assess involvement of small bowel. Findings such as full thickness inflammation, wall thickening of small bowel, strictures and fistula tract were in favor of CD. Also pelvic MRI used for evaluating perianal disease if suspected.

\section{5) Pathologic}

Biopsies were routinely obtained during endoscopy. Report of crypt abscess destruction and non-caseating granuloma were evidence of UC and CD disease respectively.

\section{Disease Activity}

Disease activity were assessed by international patient-centered instruments which were mostly based on clinical symptoms and were validated and correlated with colonoscopic, laboratory, and imaging findings. IBD-control-8 questionnaire for checking patients' recent symptoms (score more than 13 indicates inactive disease $)^{13}$ and Manitoba IBD index for checking disease control in 6 months before interview (score more than 4 indicates inactive disease) were used in IRCC questionnaire. $^{14}$

\section{Drug Availability}

More than 95\% of Iranians are covered by national health insurance and cost of almost all IBD-related drugs including biologics are supported. Furthermore, patients have access to all type of treatments including anti-TNF drugs. Therefore, the pattern of IBD treatment in Iran would not be affected by drug availability.

\section{Questionnaire}

We define our minimum data set for IRCC according to an international consensus on standard set of patient-centered outcome for IBD which has been reported recently. ${ }^{15}$ The ques- 
tionnaire has been developed and agreed following an exhaustive consultation process with technical supervisors and steering committee. And contains following sections: demographics, habitual, comorbidities, disease activity, quality of life, healthcare utilization, treatment complications, survival and disease control (Table 1, Supplementary Material 1). Standard queries were included in the questionnaire to interview patients on education level, ${ }^{16}$ past medical history, ${ }^{17}$ symptoms of IBD in last 2 weeks ${ }^{13}$ and disease control in last 6 months. ${ }^{14}$ Questions containing information on disease subtype, extent of intestinal involvement, clinical phenotype, and IBD-related surgery were specified to be answered by physician (clinicianreported). Since opium and hookah use is common in Iran, related questions were added to questionnaire in order to evaluate their association with IBD.

\section{Enrollment}

In order to enroll cases, a web-based software and website ${ }^{18}$ were designed by our IT technician. Collection of data would be performed in different ways after obtaining the consent from patients (Fig. 3). Any gastroenterologist could register his/her IBD patient information in the web-based software primarily and then the registrar from IRCC office calls the patient and fills the rest of questions. Patient enters his/her contact information directly into the web-based software. Registrar then calls the patient and fills the rest of questions. Any IRCC collaborator could fill a paper-based questionnaire and then sends it to the IRCC office by post to be entered to the database by registrars. Patient can directly refer to IRCC office and provides the necessary information to the registrar. For confidentiality of data, our software generates an identification code for each case which would be used for exporting anonymized data.

\section{Follow-up}

After enrolling IBD patients, annual telephone call will be made to each patient and data about treatment complications, symptoms and quality of life, admission and emergency department visits, and disease control will be collected. In addition, all IRCC collaborators are available to help and complete the follow up data. Any outcome such as, IBD flare-up, IBD-related surgery and disability and death will be recorded and related documents will be reviewed accordingly.

\section{Pilot Study}

To evaluate the feasibility of our project, we designed a pilot
Table 1. Variables Included in Questionnaire of the Iranian Registry of Crohn's and Colitis

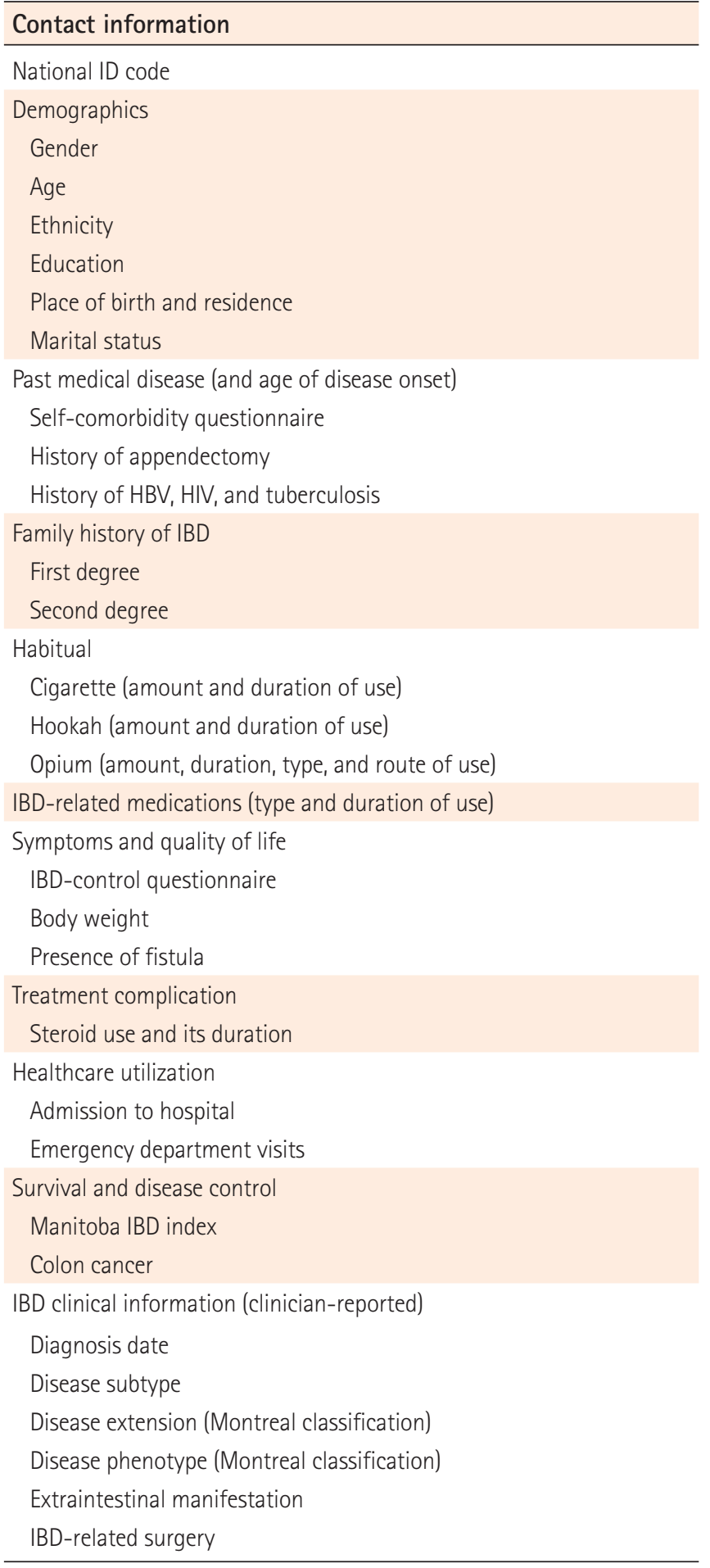

study on 553 IBD patients whom their contact information was available. The majority of studied subjects $(\mathrm{n}=529,95 \%)$ were registered primarily by their gastroenterologist with paper-based questionnaire and then the registrar from IRCC of- 


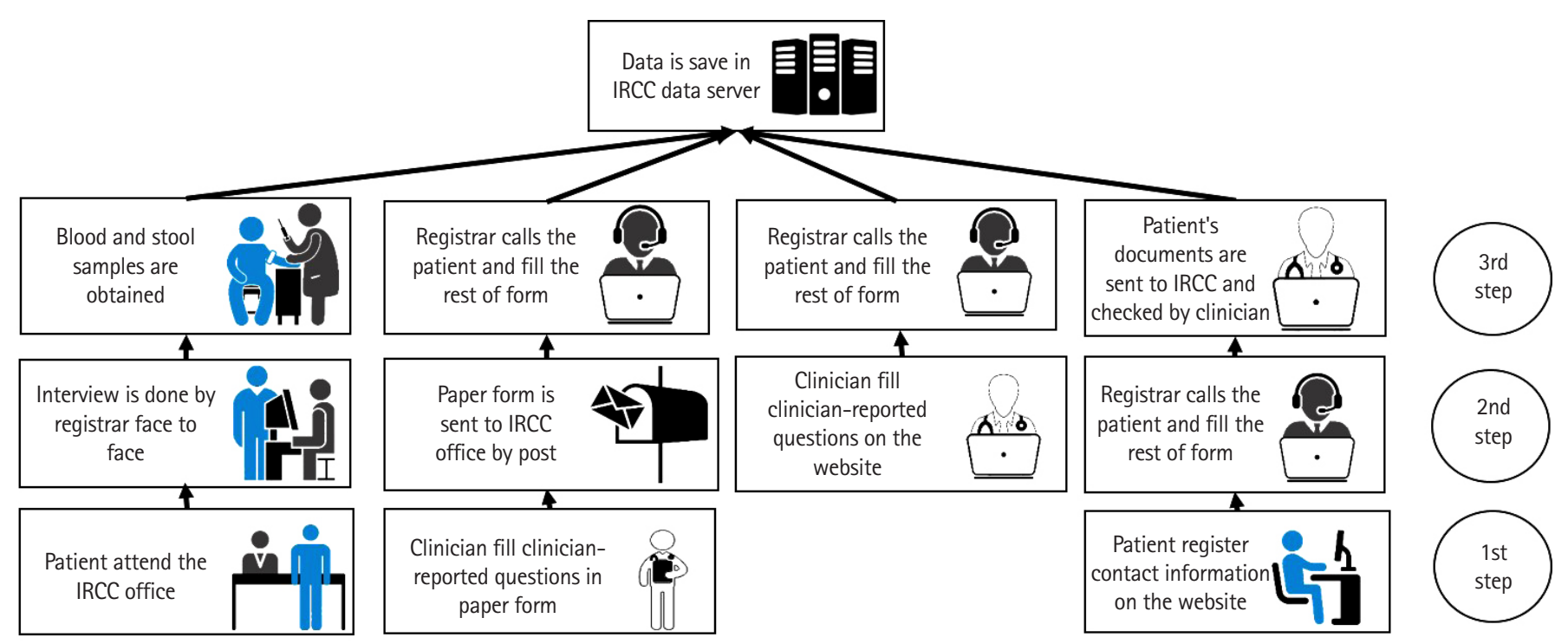

Fig. 3. Data collection pattern in Iranian Registry of Crohn's and Colitis (IRCC).

fice called them for interview. The minority of patients $(\mathrm{n}=12$, $2 \%$ ) attended the IRCC office directly and 17 patients (3\%) were registered into the website by their physician. At the end of pilot study, all IRCC collaborators were invited and the study design, data collection, questionnaire and results of pilot study were presented. All comments on study protocol were considered and the final version of questionnaire was developed. The quality control protocol for process of data collection and data accuracy was defined according to the caveats in pilot study.

\section{Quality Control of Registry Process}

Case enrollment was based on diagnosis of IRCC collaborators who were committed to use standard disease definition and protocol. Quality of data collection was monitored by random recording and checking of interviews performed by registrars. Moreover, our designed software has some validation rules which does not let wrong data to be registered and it has monitoring dashboard for executive manager to follow rate of response and missing data. All registrars were trained in the IRCC office.

\section{Quality Control of Data}

We used standard minimum data set of outcomes related to $\mathrm{IBD},{ }^{15}$ to guarantee that our data would be comparable to other IBD registries throughout the world. As IRCC collaborators were committed to share the information of eligible cases, we can assume that selection bias is minimal. We expect the com- pleteness of collected data by using the IRCC software which has obligatory fields for necessary questions. There is also a protocol for random checking of questions answered by physician in order to check the construct validity of clinician-reported questions. Annual report will be released and sent to registry office at deputy of research in ministry of health and all IRCC collaborators.

\section{Statistics}

Face validity of our questionnaire was checked by multiple sessions of expert panel for reviewing the questions and by receiving feedbacks from registrars during and after pilot study. The reliability of each section was evaluated by Cronbach's $\alpha$. We used chi-square test for comparing categorical variables and ANOVA with Bonferroni post hoc test for comparing continuous variables. $P$-value less than 0.05 considered significant. Analysis carried out using STATA software, version 11 (StataCorp LLC, College Station, TX, USA).

\section{RESULTS}

The mean age of participants was 38.60 years (SD, 12.86) with 312 of them were male (56.42\%). About half of subjects $(n=303$, $54.78 \%$ ) had university education. Fars ethnicity comprise $64.74 \%$ of our enrolled subjects $(n=358)$. Majority of registered subjects had UC $(\mathrm{n}=378,68.35 \%)$. We observed that 68 of patients (12.30\%) were smoker and 44 subjects (7.96\%) were hookah users and only 13 patients (2.35\%) reported opium 
Table 2. Characteristics of Pilot Study of the Iranian Registry of Crohn's and Colitis

\begin{tabular}{|c|c|}
\hline Characteristic & Value \\
\hline \multicolumn{2}{|l|}{ Sex } \\
\hline Male & $312(56.42)$ \\
\hline Female & 241 (43.58) \\
\hline \multicolumn{2}{|l|}{ Age group (yr) } \\
\hline $0-19$ & $21(3.80)$ \\
\hline $20-29$ & 106 (19.17) \\
\hline $30-39$ & $206(37.25)$ \\
\hline $40-49$ & $120(21.70)$ \\
\hline $50-59$ & 61 (11.03) \\
\hline $60-69$ & $27(4.88)$ \\
\hline$\geq 70$ & $12(2.17)$ \\
\hline \multicolumn{2}{|l|}{ Education } \\
\hline Illiterate & $6(1.08)$ \\
\hline Primary school & $20(3.62)$ \\
\hline Middle school & 58 (10.49) \\
\hline High school & 166 (30.02) \\
\hline Associate degree & $49(8.86)$ \\
\hline Bachelor & 170 (30.74) \\
\hline Master & 65 (11.75) \\
\hline Doctoral & $19(3.44)$ \\
\hline \multicolumn{2}{|l|}{ Depression } \\
\hline No & $500(90.42)$ \\
\hline Yes & $53(9.58)$ \\
\hline \multicolumn{2}{|l|}{ Appendectomy } \\
\hline No & 495 (89.51) \\
\hline Yes & 58 (10.49) \\
\hline \multicolumn{2}{|l|}{ Smoking } \\
\hline Never & 485 (87.7) \\
\hline Current user & $36(6.51)$ \\
\hline Past user & $32(5.79)$ \\
\hline \multicolumn{2}{|l|}{ Age (yr) } \\
\hline UC & $39.51 \pm 13.25$ \\
\hline$C D$ & $36.10 \pm 11.78$ \\
\hline Both & $38.58 \pm 12.87$ \\
\hline \multicolumn{2}{|l|}{ Hookah } \\
\hline Never & 509 (92.04) \\
\hline Current user & 32 (5.79) \\
\hline Past user & $12(2.17)$ \\
\hline \multicolumn{2}{|l|}{ Opium } \\
\hline Never & 540 (97.65) \\
\hline Current user & $9(1.63)$ \\
\hline Past user & $4(0.72)$ \\
\hline
\end{tabular}

(Continued to the next)
Table 2. Continued

\begin{tabular}{|c|c|}
\hline Characteristic & Value \\
\hline \multicolumn{2}{|l|}{ Ethnicity } \\
\hline Fars & $358(64.74)$ \\
\hline Turk & $90(16.27)$ \\
\hline Lor & $33(5.97)$ \\
\hline Kurd & $35(6.33)$ \\
\hline Arab & $7(1.27)$ \\
\hline Other & $30(5.42)$ \\
\hline \multicolumn{2}{|c|}{ Family history (1st degree) } \\
\hline UC & $50(9.04)$ \\
\hline$C D$ & $15(2.71)$ \\
\hline Both & $65(11.75)$ \\
\hline \multicolumn{2}{|c|}{ Family history (2nd degree) } \\
\hline UC & $29(5.24)$ \\
\hline$C D$ & $9(1.62)$ \\
\hline Both & $38(6.87)$ \\
\hline \multicolumn{2}{|c|}{ Disease activity during 2 weeks before enrollment ${ }^{a}$} \\
\hline Quiescent & $289(52.26)$ \\
\hline Active & $264(47.74)$ \\
\hline \multicolumn{2}{|c|}{ Disease activity during 6 months before enrollment ${ }^{b}$} \\
\hline Quiescent & $341(61.66)$ \\
\hline Active & $212(38.34)$ \\
\hline \multicolumn{2}{|c|}{ Age at diagnosis (yr) } \\
\hline UC & $31.36 \pm 12.16$ \\
\hline$C D$ & $27.92 \pm 11.32$ \\
\hline Both & $30.43 \pm 11.98$ \\
\hline
\end{tabular}

Values are presented as number (\%) or mean \pm SD.

${ }^{a}$ According to IBD-control-8 questionnaire.

${ }^{b}$ According to Manitoba IBD index.

consumption. Fifty-eight patients (10.48\%) had history of appendectomy of which 30 cases were before diagnosis of IBD. Family history were positive in 65 subjects (11.75\%) for first degree (50 relatives had UC) and 38 (6.87\%) for second-degree relatives (29 relatives had UC). The age at diagnosis for UC and CD were 31.36 (SD, 12.16) and 27.92 (SD, 11.32) years respectively $(P=0.00)$. Disease duration were similar in both subgroups of UC and CD $(8.40 \pm 7.26$ and $8.18 \pm 7.18$ years, $P=0.91)$. Most of patients $(\mathrm{n}=341,61.66 \%)$ had quiescent disease in 2 weeks before enrollment and 289 patients (52.22\%) had IBD in remission during the last 6 months (Table 2). 5-Aminosalicylate (5-ASA) and immunomodulator (azathioprine or 6-mercaptopurine or methotrexate) were currently used by $67.81 \%$ and $43.58 \%$ of participants, respectively (Table 3, Fig. 
Table 3. Pattern of IBD Treatment in Pilot Study of the Iranian Registry of Crohn's and Colitis

\begin{tabular}{lccc}
\hline & UC & CD & $\begin{array}{c}\text { IBD } \\
\text { unclassified }\end{array}$ \\
\hline Prednisolone & & & \\
Never & $135(35.71)$ & $53(32.72)$ & $8(61.54)$ \\
Currently using & $42(11.11)$ & $22(13.58)$ & $1(7.69)$ \\
Previously using & $201(53.17)$ & $87(53.70)$ & $4(30.77)$ \\
Immunomodulator & & & \\
Never & $159(42.06)$ & $32(19.75)$ & $8(61.54)$ \\
Currently using & $150(39.68)$ & $88(54.32)$ & $3(23.08)$ \\
Previously using & $69(18.25)$ & $42(25.93)$ & $2(15.38)$ \\
5-ASA & & & \\
Never & $9(2.38)$ & $19(11.73)$ & $3(23.08)$ \\
Currently using & $301(79.63)$ & $70(43.21)$ & $4(30.77)$ \\
Previously using & $68(17.99)$ & $73(45.06)$ & $6(46.15)$ \\
Anti-TNF & & & \\
Never & & & $10(76.92)$ \\
Currently using & $88(23.28)$ & $92(56.79)$ & $2(15.38)$ \\
Previously using & $57(15.08)$ & $23(14.20)$ & $1(7.69)$ \\
Duration of use (yr) & & & \\
Prednisolone & $2.63 \pm 4.40$ & $2.61 \pm 3.35$ & $2.37 \pm 3.24$ \\
Immunomodulator & $5.21 \pm 5.81$ & $5.61 \pm 5.82$ & $2.90 \pm 3.01$ \\
5-ASA & $7.09 \pm 7.22$ & $5.01 \pm 4.90$ & $4.79 \pm 4.64$ \\
Anti-TNF & $1.91 \pm 3.08$ & $2.12 \pm 2.42$ & $2.50 \pm 0.50$ \\
\hline
\end{tabular}

Values are presented as number (\%) or mean \pm SD.

5-ASA, 5-aminosalicylate.

4). The reliability of each section of questionnaire was checked and all sections had Cronbach's $\alpha>0.6$ (Table 4).

Compared to patients with UC, those with CD were treated more frequently with immunomodulator (azathioprine or 6mercaptopurine or methotrexate) $(38.36 \%$ vs. $70.99 \%, P<0.001)$ or anti-TNF (adalimumab or infliximab) (57.94\% vs. $80.25 \%$, $P<0.001)$. There was no significant difference in disease activity score between patients whom were treated with adalimumab compared to those consumed infliximab $(P=0.52)$.

\section{DISCUSSION}

In this paper, we introduced the design and implementation of IBD registry in Iran named "IRCC." Although there were few IBD registries at provincial level in Iran, ${ }^{19,20}$ to best of our knowledge, this is the first nation-wide registry running in Middle East. We have assessed the feasibility of our study and face va-
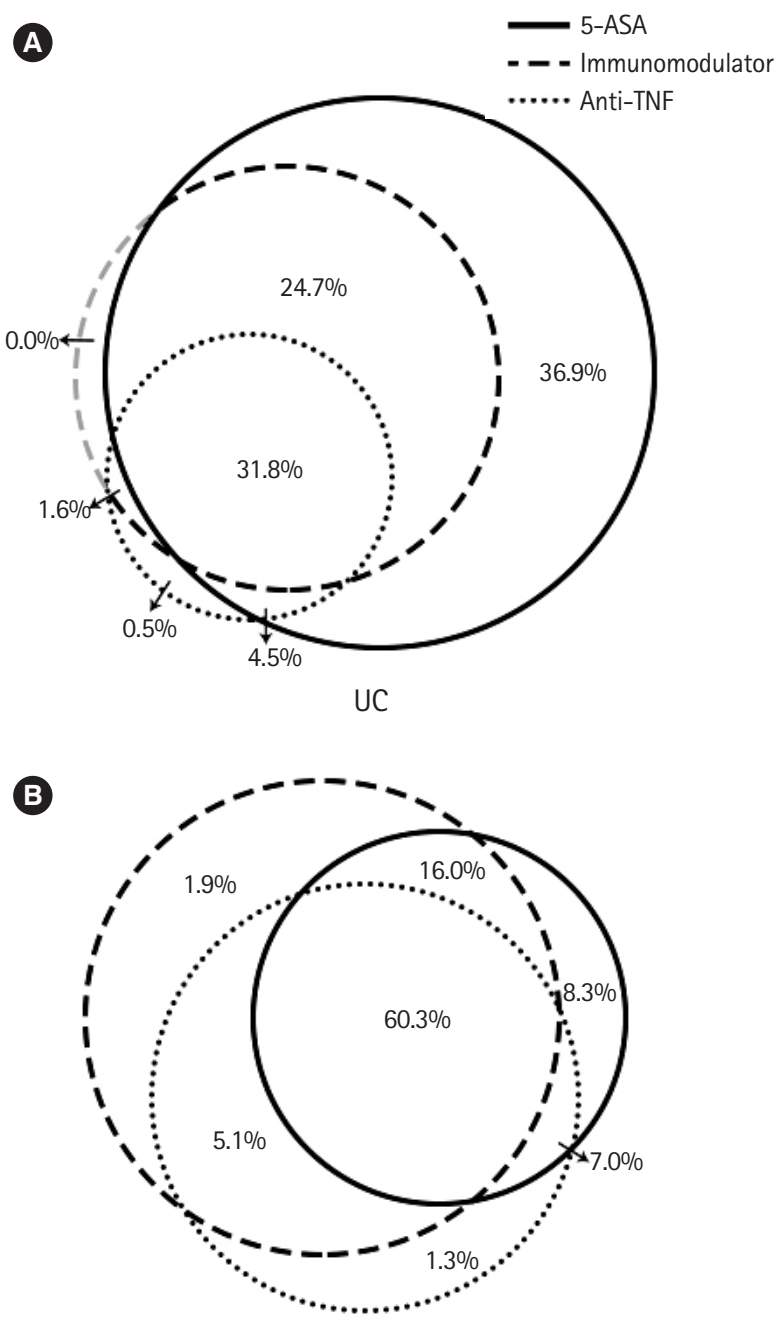

CD

Fig. 4. $(A, B)$ The percentage of previous or current drug use for treatment of $U C$ and $C D$ in pilot study of the Iranian Registry of Crohn's and Colitis. 5-ASA, 5-aminosalicylate.

Table 4. Cronbach's $\alpha$ of Different Sections of the Questionnaire in the Iranian Registry of Crohn's and Colitis

\begin{tabular}{lc}
\hline Section of questionnaire & Cronbach's $\alpha$ \\
\hline Past medical history & 0.79 \\
Habitual & \\
Cigarette & 0.76 \\
Hookah & 0.84 \\
Opium & 0.63 \\
Treatment & 0.76 \\
Symptoms and quality of life & 0.82 \\
Healthcare use and treatment complication & 0.63 \\
Survival and disease control & 0.68 \\
\hline
\end{tabular}


lidity and internal reliability of designed questionnaire by performing a pilot study on 553 subjects.

Although registry is a prospective observational study and patients are not randomly assigned to specific treatment, its result is as powerful as randomized controlled trials (RCT) or cohort studies, because patients are followed in their real-world life and are not excluded due to different study criteria. Moreover, the follow-up duration is always longer than RCTs. These advantages could help investigators to more efficiently evaluate the natural course of disease and obtain more accurate date regarding treatment response and disease survival. ${ }^{21}$

Similar to IRCC, multicenter registries are held in other part of the world. For example, SHARE registry in the United States is cooperation of 7 referral IBD centers focused mostly on biomedical researches and treatment of IBD. ${ }^{22}$ There are also multicenter IBD registries in Europe such as Austrian IBD center cohort which is comprised of 14 tertiary centers and aimed to study on disease phenotype and activity and treatment of IBD. ${ }^{23}$ In Asia, there are 2 multihospital registries held in china and 1 in Hong Kong which epidemiologic data from these registries were reported. ${ }^{24-26}$ Also there is an ongoing multicenter nation-wide IBD registry in Korea. ${ }^{27}$ Although IRCC is a multicenter IBD registry at present time, but we have planned to expand it by including all GI centers around the country. This task is more achievable with establishment of ongoing electronic medical record system in Iran. Because we would be able to use these records by IRCC software similar to other registries such as IBD UK registry ${ }^{28}$ or IBD registry at Western Pennsylvania. ${ }^{29}$

Another very important initiative is the International Inflammatory Bowel Disease Genetics Consortium which is a network of world researchers focusing mainly on the genetics of IBD. It has undertaken a number of large-scale genome-wide association studies of CD and UC, and have been able to identified more than 200 IBD risk loci with majority of these loci are shared across diverse ancestry groups, including Iranian. ${ }^{30}$ By setting up bio-bank of IBD patient in Iran further studies on IBD genetics will be possible. Also collecting blood samples will be useful for study on new biomarkers for diagnosis and monitoring IBD and colleting stool samples will help to study the role of microbiota in IBD etiology and disease activity.

There are several strengths in this study including a prospective design, using a validated and reliable questionnaire based on accepted international minimum data set, ${ }^{15}$ a well-designed software and website, flexible options to enroll IBD cases, and registering patients with their unique national ID which will allow data linkage to other national studies such as cancer registry and cause of death registry. There are also some limitations such as absence of link to patients' detail medical records at present time and before establishment of national electrical medical record in Iran.

In conclusion IRCC could become a reliable infrastructure for national and international research on IBD and at the same time improve the care of IBD patients and provide national information for policy makers to better plan for controlling IBD in Iran.

\section{FINANCIAL SUPPORT}

This work was supported by Deputy of Research of the Ministry of Health and Medical Education, and Iranian Association of Gastroenterology and Hepatology.

\section{CONFLICT OF INTEREST}

No potential conflict of interest relevant to this article was reported.

\section{AUTHOR CONTRIBUTION}

Study design, data analysis, writing the first draft, finalizing the manuscript: Malekzadeh MM. Study design and conduct, data acquisition, revising the manuscript and confirm the final version of manuscript: Sima A, Vahedi H. Study design, revising the manuscript and confirm the final version of manuscript: Alatab S, Zendedel K. Study design, data acquisition, revising the manuscript and confirm the final version of manuscript: Sadeghi A, Malekzadeh R. Data acquisition, revising the manuscript and confirm the final version of manuscript: Daryani NE, Adibi P, Maleki I, Vossoughinia H, Fakheri H, Yazdanbod A, Taghavi SA, Aghazadeh R, Somi MH. Approval of final manuscript: all authors.

\section{ACKNOWLEDGEMENTS}

We thank Digestive Disease Research Institute (DDRI) executive manager for his logistic support for setting up Iranian Registry of Crohn's and Colitis (IRCC) office. Also we thank all collaborators of IRCC who shared their cases with us. We thank Mrs. Mona Malekzadeh for her contribution to graphical works of this project. 


\section{REFERENCES}

1. Leddin D, Tamim H, Levy AR. Decreasing incidence of inflammatory bowel disease in eastern Canada: a population database study. BMC Gastroenterol 2014;14:140.

2. Molinié F, Gower-Rousseau C, Yzet T, et al. Opposite evolution in incidence of Crohn's disease and ulcerative colitis in Northern France (1988-1999). Gut 2004;53:843-848.

3. Loftus CG, Loftus EV Jr, Harmsen WS, et al. Update on the incidence and prevalence of Crohn's disease and ulcerative colitis in Olmsted County, Minnesota, 1940-2000. Inflamm Bowel Dis 2007;13:254-261.

4. Ng SC, Shi HY, Hamidi N, et al. Worldwide incidence and prevalence of inflammatory bowel disease in the 21st century: a systematic review of population-based studies. Lancet 2018; 390:2769-2778.

5. Prideaux L, Kamm MA, De Cruz PP, Chan FK, Ng SC. Inflammatory bowel disease in Asia: a systematic review. J Gastroenterol Hepatol 2012;27:1266-1280.

6. Lakatos L, Lakatos PL. Is the incidence and prevalence of inflammatory bowel diseases increasing in Eastern Europe? Postgrad Med J 2006;82:332-337.

7. Ouyang Q, Tandon R, Goh KL, Ooi CJ, Ogata H, Fiocchi C. The emergence of inflammatory bowel disease in the Asian Pacific region. Curr Opin Gastroenterol 2005;21:408-413.

8. Malekzadeh MM, Vahedi H, Gohari K, et al. Emerging epidemic of inflammatory bowel disease in a middle income country: a nation-wide study from Iran. Arch Iran Med 2016;19:215.

9. Leung WK. Optimization of inflammatory bowel disease cohort studies in Asia. Intest Res 2015;13:208-212.

10. Mojarrab S, Rafei A, Akhondzadeh S, Jeddian A, Jafarpour M, Zendehdel K. Diseases and health outcomes registry systems in I.R. Iran: successful initiative to improve public health programs, quality of care, and biomedical research. Arch Iran Med 2017;20:696-703.

11. Bernstein CN. Large registry epidemiology in IBD. Inflamm Bowel Dis 2017;23:1941-1949.

12. Bernstein CN, Eliakim A, Fedail S, et al. World gastroenterology organisation global guidelines inflammatory bowel disease: update August 2015. J Clin Gastroenterol 2016;50:803818.

13. Bodger K, Ormerod C, Shackcloth D, Harrison M; IBD Control Collaborative. Development and validation of a rapid, generic measure of disease control from the patient's perspective: the IBD-control questionnaire. Gut 2014;63:1092-1102.
14. Clara I, Lix LM, Walker JR, et al. The Manitoba IBD Index: evidence for a new and simple indicator of IBD activity. Am J Gastroenterol 2009;104:1754-1763.

15. Kim AH, Roberts C, Feagan BG, et al. Developing a standard set of patient-centred outcomes for inflammatory bowel disease-an international, cross-disciplinary consensus. J Crohns Colitis 2018;12:408-418.

16. International Standard Classification of Education: ISCED 2011. UNESCO Digital Library Web site. https://unesdoc.unesco.org/ark:/48223/pf0000219109. Accessed March 6, 2019.

17. Sangha O, Stucki G, Liang MH, Fossel AH, Katz JN. The SelfAdministered Comorbidity Questionnaire: a new method to assess comorbidity for clinical and health services research. Arthritis Rheum 2003;49:156-163.

18. National Network for Research on IBD. http://www.ibd-info.ir. Accessed April 18, 2019.

19. Taghavi SA, Bagheri Lankarani K, Moini M, Hamidpour L, Ardebili M, Mansoorabadi Z. Establishment of a population-based registry of inflammatory bowel diseases in Fars Province, Iran. Middle East J Dig Dis 2012;4:97-101.

20. Sherkat R, Rostami S, Yaran M, et al. Establishment and development of the first biobank of inflammatory bowel disease, suspected to primary immunodeficiency diseases in Iran. Adv Biomed Res 2018;7:45.

21. Bergqvist D, Björck M, Säwe J, Troëng T. Randomized trials or population-based registries. Eur J Vasc Endovasc Surg 2007; 34:253-256.

22. Kochar B, Long MD, Galanko J, Raffals LE, Ananthakrishnan A, Sandler RS. Inflammatory bowel disease is similar in patients with older onset and younger onset. Inflamm Bowel Dis 2017;23:1187-1194.

23. Novacek G, Weltermann A, Sobala A, et al. Inflammatory bowel disease is a risk factor for recurrent venous thromboembolism. Gastroenterology 2010;139:779-787.

24. Ng SC, Leung WK, Shi HY, et al. Epidemiology of inflammatory bowel disease from 1981 to 2014: results from a territorywide population-based registry in Hong Kong. Inflamm Bowel Dis 2016;22:1954-1960.

25. Zeng Z, Zhu Z, Yang Y, et al. Incidence and clinical characteristics of inflammatory bowel disease in a developed region of Guangdong Province, China: a prospective population-based study. J Gastroenterol Hepatol 2013;28:1148-1153.

26. Zhao J, Ng SC, Lei Y, et al. First prospective, population-based inflammatory bowel disease incidence study in mainland of China: the emergence of "western" disease. Inflamm Bowel Dis 2013;19:1839-1845. 
27. Cheon JH, Kim YS, Ye BD, et al. Crohn's Disease Clinical Network and Cohort (CONNECT) Study: the first step toward nationwide multicenter research of Crohn's disease in Korea. Intest Res 2014;12:173-175.

28. The UK IBD registry. IBD Registry Web site. https://ibdregistry.org.uk/. Accessed March 6, 2019.

29. Anderson AJ, Click B, Ramos-Rivers C, et al. Development of an inflammatory bowel disease research registry derived from observational electronic health record data for comprehensive clinical phenotyping. Dig Dis Sci 2016;61:3236-3245.

30. Liu JZ, van Sommeren S, Huang H, et al. Association analyses identify 38 susceptibility loci for inflammatory bowel disease and highlight shared genetic risk across populations. Nat Genet 2015;47:979-986. 
See "Iranian Registry of Crohn's and Colitis: study profile of first nation-wide inflammatory bowel disease registry in Middle East" on page 330-339.

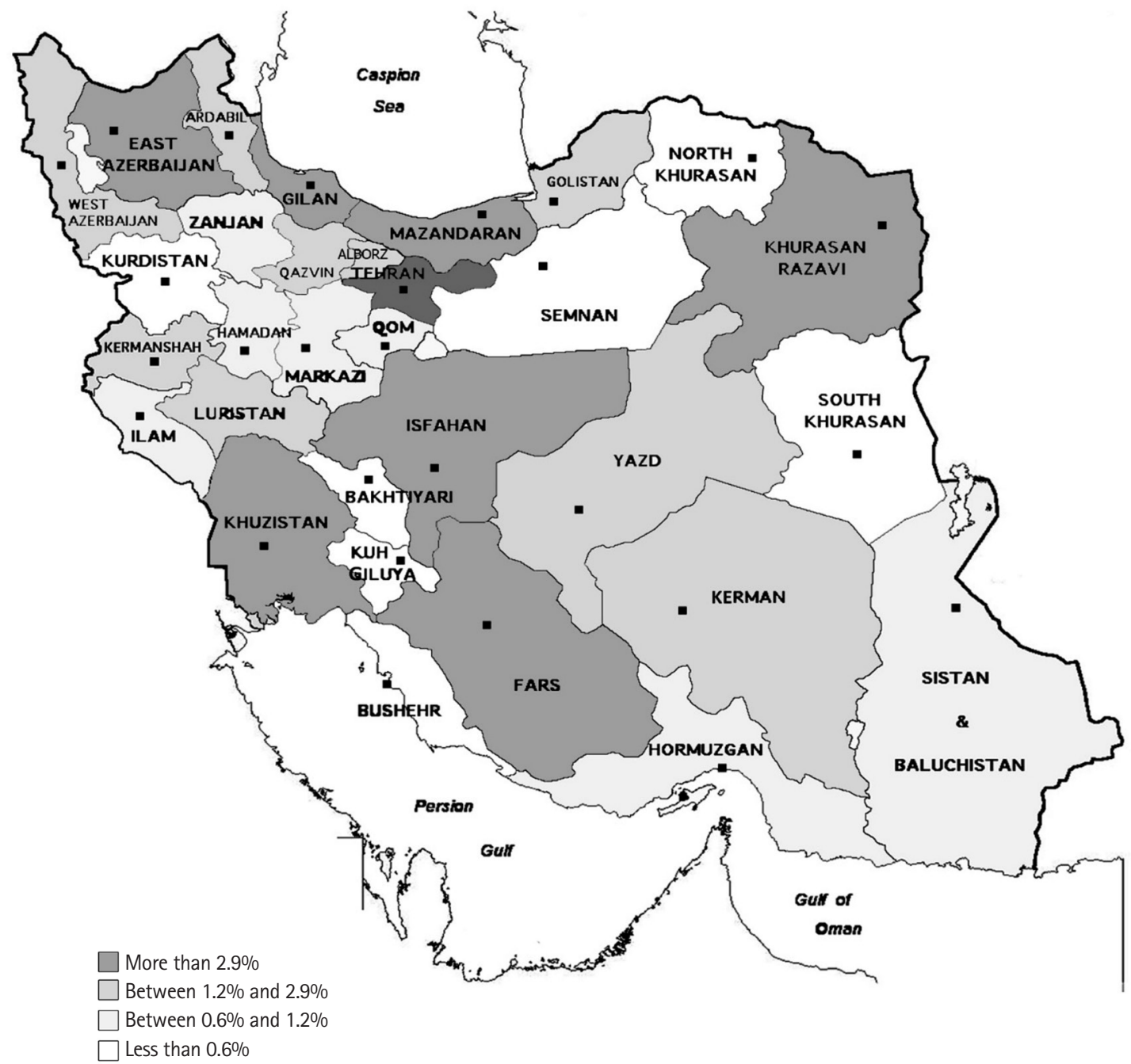

Supplementary Fig. 1. Percentage of gastroenterologists work in different provinces of Iran. 
Supplementary Table 1. Distribution of Gastroenterologists in Iran and Participation Rate of Gastroenterologists of Different Provinces with IRCC Study

\begin{tabular}{|c|c|c|c|c|}
\hline Province & $\begin{array}{c}\text { No. of gastroenterologist } \\
\text { by IRCC list }\end{array}$ & $\begin{array}{c}\text { No. of gastroenterologist } \\
\text { by IAGH list }\end{array}$ & $\begin{array}{l}\text { Participation } \\
\text { rate }\end{array}$ & $\begin{array}{c}\text { Percent of } \\
\text { gastroenterologist }\end{array}$ \\
\hline Alborz & 2 & 9 & 22.2 & 1.74 \\
\hline Ardabil & 6 & 6 & 100 & 1.16 \\
\hline Azarbaijan East & 15 & 19 & 78.9 & 3.68 \\
\hline Azarbaijan West & 8 & 9 & 88.9 & 1.74 \\
\hline Bakhtiyari & 2 & 2 & 100 & 0.39 \\
\hline Bushehr & 2 & 2 & 100 & 0.39 \\
\hline Fars & 30 & 30 & 100 & 5.80 \\
\hline Gilan & 14 & 19 & 73.7 & 3.68 \\
\hline Golistan & 6 & 6 & 100 & 1.16 \\
\hline Hamedan & 3 & 5 & 60.0 & 0.97 \\
\hline Hormuzgan & 4 & 4 & 100 & 0.77 \\
\hline Ilam & 3 & 4 & 75.0 & 0.77 \\
\hline Isfahan & 26 & 36 & 72.2 & 6.96 \\
\hline Kerman & 8 & 8 & 100 & 1.55 \\
\hline Kermanshah & 5 & 6 & 83.3 & 1.16 \\
\hline Khurasan North & 1 & 1 & 100 & 0.19 \\
\hline Khurasan Razavi & 34 & 42 & 80.9 & 8.12 \\
\hline Khurasan South & 1 & 1 & 100 & 0.19 \\
\hline Khuzistan & 11 & 15 & 73.3 & 2.90 \\
\hline Kohgiluya & 2 & 2 & 100 & 0.39 \\
\hline Kurdistan & 2 & 2 & 100 & 0.39 \\
\hline Luristan & 4 & 6 & 66.7 & 1.16 \\
\hline Markazi & 3 & 3 & 100 & 0.58 \\
\hline Mazandaran & 18 & 30 & 60.0 & 5.80 \\
\hline Qazvin & 6 & 6 & 100 & 1.16 \\
\hline Qom & 5 & 5 & 100 & 0.97 \\
\hline Semnan & 2 & 2 & 100 & 0.39 \\
\hline Sistan \& Baluchistan & 2 & 3 & 66.7 & 0.58 \\
\hline Tehran & 215 & 219 & 98.2 & 42.36 \\
\hline Yazd & 7 & 11 & 63.6 & 2.13 \\
\hline Zanjan & 2 & 4 & 50.0 & 0.77 \\
\hline Total & 449 & 517 & 86.8 & 100 \\
\hline
\end{tabular}

IRCC, Iranian Registry of Crohn's and Colitis; IAGH, Iranian Association of Gastroenterology and Hepatology. 


\section{IRCC enrollment questionnaire}

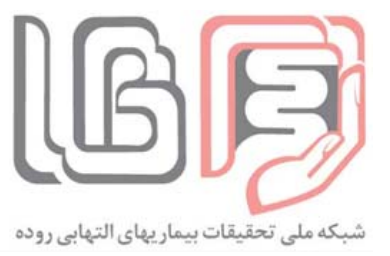

\section{Patient-reported questions:}

\section{Patient Demographic data}

1. Date of filling questionnaire:

2. First Name:

3. Last Name:

4. National code:

5. Gender: 1) Male 2) Female

6. Marital status: 1) Single 2) Married

7. Landline Phone:

8. Mobile Phone:

9. Birth Date:

10. Birth province:

11. Birth city:

12. Living province:

13. Living city:

14. Education: 0) illiterate 1) Primary school 2 2) Middle school 3) High school 4) Associate degree 5) Bachelor 6) Master 7) Doctoral
15. Ethnicity: 1) Fars
2) Turk
3) Lur 4) Kord
6) Arab
7) Turkman 8) Other
16. Twin: 0) No
1) Yes
17. Twin type: 1) Homozygote $\quad$ 2) Hetrozygote
18. Your doctor name:

\section{Past medical History}

19. Did you have heart disease? $\mathrm{Y} / \mathrm{N}$

20. Did you take medication for it? $\mathrm{Y} / \mathrm{N}$

21. Did it limit your activity? $\mathrm{Y} / \mathrm{N}$

22. At what age did you get heart disease?

23. Did you have hypertension? $\mathrm{Y} / \mathrm{N}$

24. Did you take medication for it? $\mathrm{Y} / \mathrm{N}$

25. Did it limit your activity? $\mathrm{Y} / \mathrm{N}$

26. At what age did you get hypertension?

27. Did you have lung disease? $\mathrm{Y} / \mathrm{N}$

28. Did you take medication for it? $\mathrm{Y} / \mathrm{N}$

29. Did it limit your activity? $\mathrm{Y} / \mathrm{N}$

30. At what age did you get lung disease? 
31. Did you have diabetes? $\mathrm{Y} / \mathrm{N}$

32. Did you take medication for it? $\mathrm{Y} / \mathrm{N}$

33. Did it limit your activity? $\mathrm{Y} / \mathrm{N}$

34. At what age did you get diabetes?

35. Did you have gastric ulcer? $\mathrm{Y} / \mathrm{N}$

36. Did you take medication for it? $\mathrm{Y} / \mathrm{N}$

37. Did it limit your activity? $\mathrm{Y} / \mathrm{N}$

38. At what age did you get gastric ulcer?

39. Did you have kidney disease? $\mathrm{Y} / \mathrm{N}$

40. Did you take medication for it? $\mathrm{Y} / \mathrm{N}$

41. Did it limit your activity? $\mathrm{Y} / \mathrm{N}$

42. At what age did you get kidney disease?

43. Did you have liver disease? $\mathrm{Y} / \mathrm{N}$

44. Did you take medication for it? $\mathrm{Y} / \mathrm{N}$

45. Did it limit activity? $\mathrm{Y} / \mathrm{N}$

46. At what age did you get liver disease?

47. Did you have anemia or any other blood disease? $\mathrm{Y} / \mathrm{N}$

48. Did you take medication for it? $\mathrm{Y} / \mathrm{N}$

49. Did it limit your activity? $\mathrm{Y} / \mathrm{N}$

50. At what age did you get anemia or any other blood disease?

51. Did you had cancer? $\mathrm{Y} / \mathrm{N}$

52. Did you take medication for it? $\mathrm{Y} / \mathrm{N}$

53. Did it limit your activity? $\mathrm{Y} / \mathrm{N}$

54. At what age did you get cancer?

55. Did you have depression? $\mathrm{Y} / \mathrm{N}$

56. Did you take medication for it? $\mathrm{Y} / \mathrm{N}$

57. Did it limit your activity? $\mathrm{Y} / \mathrm{N}$

58. At what age did you get depression?

59. Did you have back pain? $\mathrm{Y} / \mathrm{N}$

60. Did you take medication for it? $\mathrm{Y} / \mathrm{N}$

61. Did it limit your activity? $\mathrm{Y} / \mathrm{N}$

62. At what age did you get back pain?

63. Did you have history of previous TB? Y/N

64. At what age did you get TB?

65. Did you have history of previous HBV? Y/N

66. At what age did you get HBV?

67. Did you have history of previous HIV? Y/N

68. At what age did you get HIV?

69. Did you have history of Appendectomy? Y/N

70. At what age did you appendectomy?

\section{Social History}

71. Do you smoke? 1) Never used 2 2) Currently using $\quad$ 3) Previously using

72. If currently or previously using, how many packet did you use per day?

73. If currently or previously using, at what age did you start smoking? 
74. If previously using, at what age did you stop smoking?

75. Have you ever used hookah? 1) Never used 2) Currently using 3 3) Previously using

76. If currently or previously using, how many days per week did you use hookah?

77. If currently or previously using, at what age did you start hookah use?

78. If currently or previously using, at what age did you stop hookah use?

79. Have you ever used opium?

$\begin{array}{llll}\text { 1) Never used } & \text { 2) Currently using } & \text { 3) Previously using }\end{array}$

80. If currently or previously using, how many days per week did you use opium?
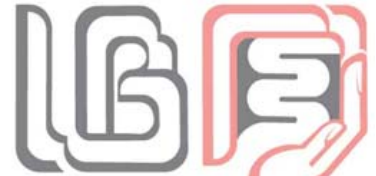

81. If currently or previously using, at what age did you start opium use?

82. If previously used, at what age did you stop opium use?

83. If currently or previously using, how many nokhod per day:

84. Type of used opium: 1) Teriak $\quad$ 2) Shireh 3 3) Sukhteh

85. Rout of administration: 1) Oral 2) Inhaled 3) Both

\section{Family History}

86. Is there history of IBD in your family members: $\mathrm{Y} / \mathrm{N}$

87. If yes, which family member: 1) father 2) mother 3) brother 4) sister 7) son 8) daughter 9) second degree

88. If yes, what was the type: 1) UC 2) CD 3) Not known which subtype

Symptoms and Signs, function and quality of life

89. Do you believe that your IBD has been well controlled in the past 2 weeks?

2) Yes 0) No 1) Not sure

90. Do you believe that your current treatment is useful in controlling your IBD?

2) Yes 0) No 1) Not sure

91. Over the past 2 weeks, have your bowel symptoms been getting worse, getting better or not changed? 2) Better 1) No change 0) Worse

In the past 2 weeks, did you:

92. Miss any planned activities because of IBD? 0) Yes 2 ) No 1 1) Not sure

93. Wake up at night because of symptoms of IBD? 0) Yes 2) No 1) Not sure

94. Suffer from significant pain or discomfort? 0) Yes 2) No 1 1) Not sure

95. Often feel lacking in energy? 0) Yes 2) No 1) Not sure

96. Feel anxious or depressed because of your IBD? 0) Yes 2) No 1) Not sure

97. Think you needed a change to your treatment? 0) Yes 2) No 1 ) Not sure

98. Weight (in kilogram):

99. Did you have fistula? 1) No 2 2) Yes 9 9) N/A

\section{Treatment Data}

100. Have you ever used prednisolone for IBD treatment?

1) Never used 2) Currently using 3) Previously using

101. When did you start prednisolone?

102. When did you finish prednisolone?

103. Who stop the treatment? 1) Patient 2) Physician 
104. If your physician stopped the treatment, what was the reason?

1) No response 2) Non-compliance 3) Recovery 4) Drug reaction 9) N/A

105. Have you ever used immunomodulator for IBD treatment?

1) Never used 2) Currently using 3)Previously using

106. What type of immunomodulator did you use?

1) Azathioprine 2) 6-Mercaptopurin 3) Methotrexate

107. When did you start immunomodulator?

108. When did you finish immunomodulator?

109. Who did stop the treatment? 1)Patient 2) Physician

110. If your physician stopped the treatment, what was the reason?

1) No response 2) Non-compliance 3) Recovery 4) Drug reaction 9) N/A

111. Have you ever used 5-ASA for IBD treatment?

1) Never used 2) Currently using 3)Previously using

112. What type of 5-ASA did you use? 1) Sulfasalazine 2) Mesalazine

113. When did you start 5-ASA?

114. When did you finish 5-ASA?

115. Who did stop the treatment? 1)Patient 2)Physician

116. If your physician stopped the treatment what was the reason?

1) No response 2) Non-compliance 3) Recovery 4) Drug reaction 9) N/A

117. Have you ever used anti-TNF for IBD treatment?

1) Never used 2) Currently using 3) Previously using

118. What type of anti-TNF did you use? 1) Infliximab 2) Adalimumab

119. When did you start anti-TNF?

120. When did you finish anti-TNF?

121. Who did stop the treatment? 1) Patient 2) Physician

122. If your physician stopped the treatment what was the reason?

1) No response 2) Non-compliance 3) Recovery 4) Drug reaction 9) N/A

\section{Healthcare utilization \& Treatment complication}

123. What was the total number of emergency room visits in past 12 months?

124. How many days was the IBD-related admissions in past 12 months?

125. Did you use steroid in previous 12 months ago? $\mathrm{Y} / \mathrm{N}$

126. Was the duration of steroid use more than 3 months? $\mathrm{Y} / \mathrm{N}$

127. Was there any admission after 3 months of starting treatment? $\mathrm{Y} / \mathrm{N}$

\section{Survival and Disease control}

128. Was your IBD in remission in last 6 months?

In the last 6 months, my disease has been

$0=$ Constantly active, giving me symptoms every day

$1=$ Often active, giving me symptoms most days

2 = Sometimes active, giving me symptoms on some days [for instance 1-2 days/week]

3 = Occasionally active, giving me symptoms $1-2$ days/month

$4=$ Rarely active, giving me symptoms on a few days in the past 6 months 
$5=$ I was well in the past 6 months: what I consider a remission or absence of symptoms

129. Did you have colorectal cancer after suffering from IBD? $\mathrm{Y} / \mathrm{N}$

130. What was the diagnosis date of colorectal cancer?

\section{Clinician-reported questions}

\section{General clinical Data}

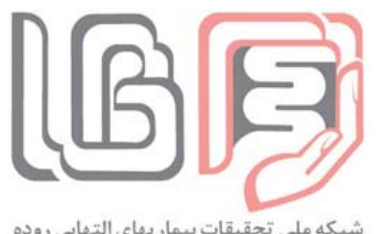

1. Date of Diagnosis:

2. Final Diagnosis: 1) UC 2) CD 3) IBD unclassified

3. If UC what is disease extent: 1) Proctitis 2) Left sided colitis 3) Pancolitis 9) N/A

4. If Crohn's what is disease location: 1) lleal 2) Colonic 3) lleocolonic 4) Isolated upper GI 9) N/A

5. If Crohn's what is disease behavior: 1) Non-fistulizing 2) Fistulizing 3 3) Stricturing 9) N/A

6. Are there any extra-intestinal manifestations?

1)Primary sclerosing cholangitis 2) Autoimmune hepatitis 3) Gallbladder stone 4) Uveitis 5) Erythema nodosum 6) Pyoderma gangrenous 7) Peripheral arthritis 8) Ankylosing spondylitis 9) N/A

\section{Surgery data}

7. Did you have surgery due to IBD disease? $\mathrm{Y} / \mathrm{N}$

8. If UC, what type of surgery have you done?

1) Proctocolectomy with ileal pouch-anal anastomosis 2) Total abdominal colectomy with

ileorectal anastomosis 3) Total abdominal colectomy with end ileostomy 9) N/A

9. If $C D$, what type of surgery have been done?
1) Resection
2) Fistulectomy and Abscess drainage
9) $N / A$ 